\title{
The optimal driving waveform for overdamped, adiabatic rocking ratchets
}

\author{
S J Lade \\ Nonlinear Physics Centre, Research School of Physical Sciences and Engineering, \\ Canberra ACT 0200, Australia \\ E-mail: steven.lade@anu.edu.au
}

Received 23 April 2008, in final form 12 May 2008

Published 16 June 2008

Online at stacks.iop.org/JPhysA/41/275103

\begin{abstract}
The optimal driving waveform among a wide class of admissible functions for an overdamped, adiabatic rocking ratchet is shown to be dichotomous. 'Optimum' is defined as that which achieves the maximum (or minimum negative) average particle velocity. Implications for the design of ratchets, for example in nanotechnological transport, may follow. The main result is applicable to a general class of adiabatic responses. Much scope exists for further studies of ratchet waveform optimization in other regimes.
\end{abstract}

PACS numbers: $05.60 . \mathrm{Cd}, 02.30 .-\mathrm{f}$

\section{Introduction}

Though its history stretches back to Feynman [1] and Smoluchowski [2], the ratchet concepta non-zero particle current arising due to symmetry breaking [3, 4] of a potential or force-has experienced a resurgence of interest in the last 15 years. Initially, the interest was motivated by the possibility of the ratchet concept explaining the operation of biological molecular motors [5-7]. Ratchets have now been realized in a wide range of physical systems [8], with possible applications in nanotechnology [9], and are being explored in quantum [10] as well as classical regimes.

Most authors choose a potential and a force that preserve or break the relevant symmetries, and then investigate the dependence of the average particle velocity, or 'ratchet current', on scalar parameters, such as damping coefficient or temperature. A few have considered a parametrized potential or force, such as a sawtooth potential with variable up-slope and downslope gradients [11], and then vary those parameters. Another such parametrization is of the amplitudes and phase of a biharmonic force, as will be discussed in section 4 .

This paper considers the task of optimizing the 'shape', or functional form, of the symmetry-breaking force or potential over all admissible functions, to achieve maximum ratchet current. That is, 'optimal' is here that which maximizes (or minimizes) ratchet current, 
not (necessarily) ratchet efficiency, Péclet number, stall force or other measures of ratchet performance [12]. Previous discussions of ratchet optimization have generally been in an abstract thermodynamic sense, remarking that maximum efficiency of a Brownian ratchet is achieved in the limit of tight coupling [13].

It is unlikely that one shape will be optimal for all regimes: overdamped, underdamped and Hamiltonian; fast and adiabatic driving; coupling through rocking and pulsating. One specific regime is here considered, this being an overdamped, adiabatic rocking ratchet, with equation of motion

$$
\eta \dot{x}(t)=-V^{\prime}(x)+f(t)+\xi(t) .
$$

This models an overdamped particle with position $x(t)$ and damping coefficient $\eta$ moving in a potential $V(x)$, subject to a force $f(t)$ and Brownian noise $\xi(t)$. In the adiabatic limit, the average velocity of the particle in the ratchet is [8]

$$
\begin{aligned}
\langle\dot{x}\rangle & =\frac{1}{T_{f}} \int_{0}^{T_{f}} v(f(t)) d t \\
v(y) & \equiv \frac{L k_{B} T\left[1-\mathrm{e}^{-L y / k_{B} T}\right]}{\eta \int_{0}^{L} \mathrm{~d} x \int_{x}^{x+L} \mathrm{~d} z \exp \left\{[V(z)-V(x)-(z-x) y] / k_{B} T\right\}},
\end{aligned}
$$

where the periods of the force and potential are $T_{f}$ and $L$, respectively, and the temperature of the Brownian noise is $k_{B} T$. 'Adiabatic' in this context means that the frequency of the driving is slow compared to any other characteristic frequencies of the system, such as the motion of the particle in its potential $V(x)$.

The potential is here set to the symmetrical $V(x)=V \cos (2 \pi x / L)$, so that a ratchet current arises from temporal symmetry breaking only, and the remaining parameters are scaled so that $V=1$ and $L=2 \pi$. In all calculations in this document $\eta=1$; any other value, as can be seen from (2), will only scale the velocity $\langle\dot{x}\rangle$. Plots of the resulting function $v(y)$ are shown in figure 1 . At large $y, v(y) \rightarrow y$; differences are only at small $y$, and even these variations disappear as $k_{B} T$ becomes large.

The optimal force, as will be proved in section 2, is dichotomous, that is, it takes only two values, switching instantaneously between them. This result holds for the optimization of any function $v(y)$ for the measure (1) over the admissibility criteria presented in that section. Using specific properties of the response function (2), section 3 finds further properties of the optimal force for that response function. Section 4 presents numerical illustrations and general discussion, followed by conclusions and directions for future work in section 5 .

\section{The optimal force is dichotomous}

The class of admissible functions $f(t)$ over which the optimization will take place is the set of (i) piecewise continuous functions with a finite number of discontinuities ('almost continuous'), which (ii) have period $T_{f}$, that is, $f\left(t+T_{f}\right)=f(t)$, are (iii) bounded, that is, there exists an $L$ for which $|f(t)| \leqslant L$ for all $t$, and (iv) have zero mean, $\int_{0}^{T_{f}} f(t) d t=0$.

Theorem 1. If force( $s)$ exist that optimize the current

$$
\langle\dot{x}\rangle(f) \equiv \frac{1}{T_{f}} \int_{0}^{T_{f}} v(f(t)) d t,
$$

then one such optimal force is dichotomous, that is, for all $t, f(t)=M$ or $N$, for some $M$ and $N$. The adiabatic response function $v(y)$ is assumed to have $v(0)=0$. 


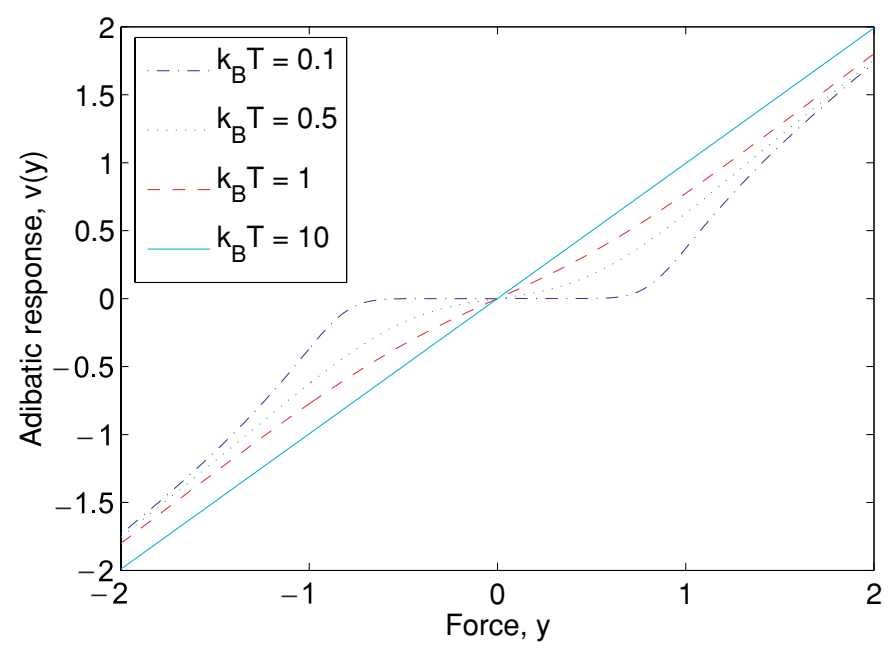

Figure 1. Adiabatic response function $v(y)$ for a range of temperatures $k_{B} T$, with $V(x)=\cos x$ and $\eta=1$.

(This figure is in colour only in the electronic version)

An 'optimum' force is defined as a force $f^{*}$ with $\langle\dot{x}\rangle\left(f^{*}\right) \geqslant\langle\dot{x}\rangle(f)$ for maximization or $\langle\dot{x}\rangle\left(f^{*}\right) \leqslant\langle\dot{x}\rangle(f)$ for minimization, where $f$ is any admissible force.

The adiabatic response function (2) satisfies $v(0)=0$.

Proof. First consider a constant force. This, by the constraint of zero mean, must have value zero, which gives zero velocity, and is therefore not optimal. Therefore any optimal force must take two (dichotomous) or more values.

Choose a small discretization size $d t$ to discretize an arbitrary admissible force $f(t)$ into a series of segments on $n d t \leqslant t<(n+1) d t, n=0, \ldots, T_{f} / d t-1$. Let $f_{i}$ refer to the discretization of $f(t)$ at $t_{i}$. Consider an admissible function which is not constant and not dichotomous, that is, it takes three or more values. For sufficiently small $d t$ there must exist three $t_{i}, i=1,2,3$ with $f_{i} \neq f_{j}$ for $i \neq j$, that is, the force takes on at least three different values. The contribution of the three intervals to the velocity is

$$
\left[v\left(f_{1}\right)+v\left(f_{2}\right)+v\left(f_{3}\right)\right] \frac{d t}{T_{f}} .
$$

Consider the effect on the velocity of replacing this three-valued section with just two values. Suppose $f_{3}$ is to be eliminated. This procedure is illustrated in figure 2 . Let the combined lengths of the intervals at which $f(t)$ will be set to $f_{1}$ and $f_{2}$ be $d t_{1}^{\prime}$ and $d t_{2}^{\prime}$, respectively, where $d t_{1}^{\prime}+d t_{2}^{\prime}=3 d t$. For $\int_{0}^{T_{f}} f(t) d t$ to remain zero,

$$
\left(f_{1}+f_{2}+f_{3}\right) d t=f_{1} d t_{1}^{\prime}+f_{2} d t_{2}^{\prime} .
$$

At least one $f_{i}$ must be greater than the average value $\left(f_{1}+f_{2}+f_{3}\right) / 3$ and at least one less than the average value. If, without loss of generality, these are labelled as $f_{1}$ and $f_{2}$, respectively, then a solution $d t_{1}^{\prime}, d t_{2}^{\prime}$ to (4) can always be found. Using (4), the change in velocity due to the change from a trichotomous to a dichotomous region can be shown to be

$$
\Delta\langle\dot{x}\rangle=\left(d t / T_{f}\right)\left(f_{3}-f_{2}\right)\left(m_{21}-m_{23}\right),
$$




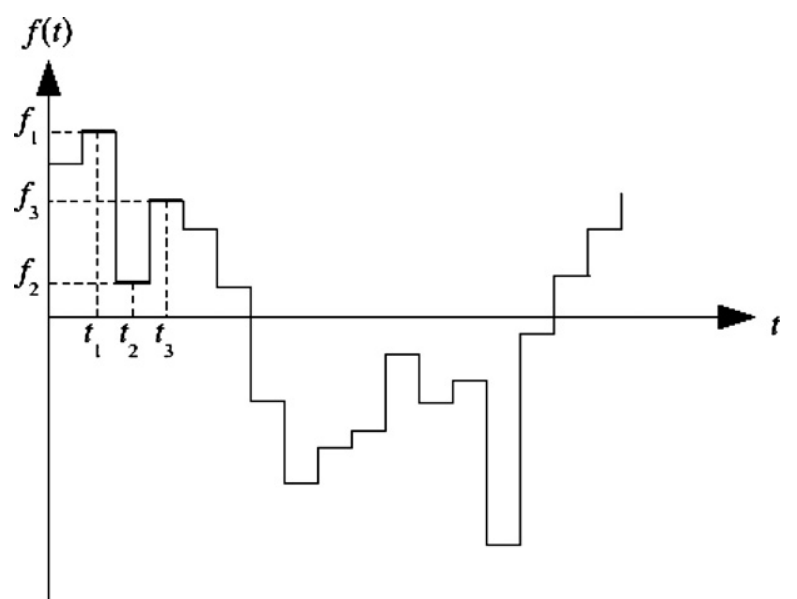

(a)

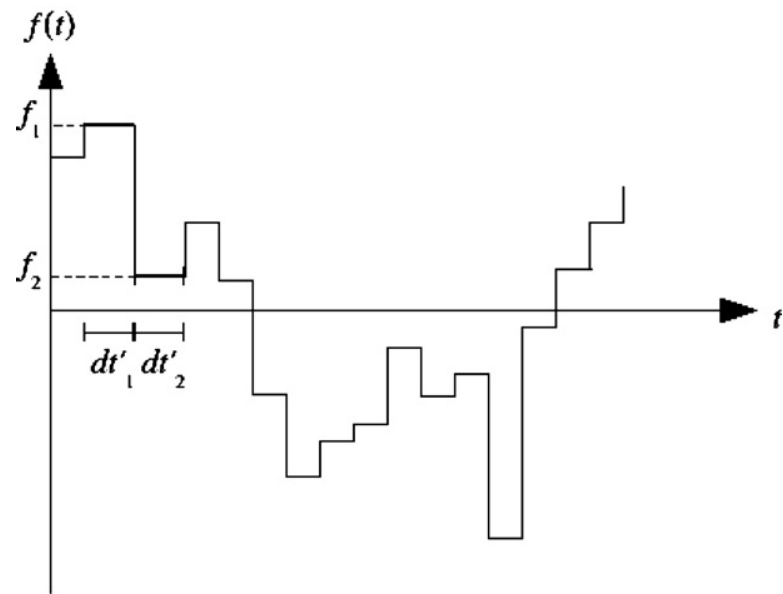

$(b)$

Figure 2. Schematic of the method to convert a trichotomous region of the discretized force into a dichotomous region. Having chosen the three values $f_{1,2,3}$ in $(a)$, the values of the force at those times are replaced with just $f_{1}$ and $f_{2}$ in $(b)$. In total, the force takes values $f_{1,2}$ for total times $d t_{1,2}^{\prime}$, respectively.

where

$$
m_{i j} \equiv \frac{v\left(f_{j}\right)-v\left(f_{i}\right)}{f_{j}-f_{i}}
$$

is the gradient of the line joining $\left(f_{i}, v\left(f_{i}\right)\right)$ and $\left(f_{j}, v\left(f_{j}\right)\right)$.

Each of the four possible arrangements of the points $\left(f_{i}, v\left(f_{i}\right)\right)$ illustrated in figure 3 will now be considered in turn.

Case illustrated by figure 3(a). In the collinear case there will be no change in velocity, $\Delta\langle\dot{x}\rangle=0$.

Case illustrated by figure $3(b)$. Without loss of generality the points may be labelled as shown. The middle point will therefore be 'removed'. As can be seen in the figure, $m_{21}>m_{23}$, and since $f_{3}>f_{2}$ then from (5), $\Delta\langle\dot{x}\rangle>0$. 


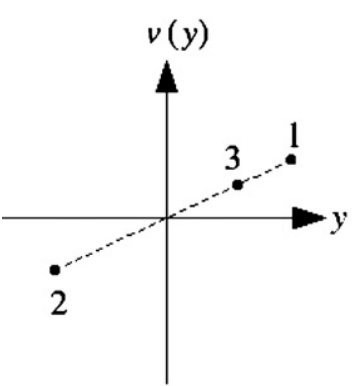

(a)

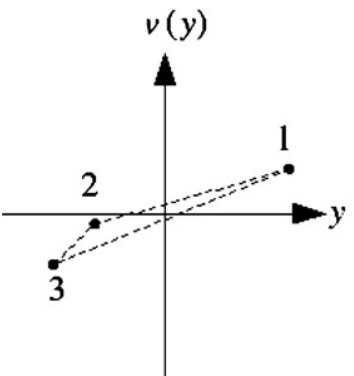

(c)

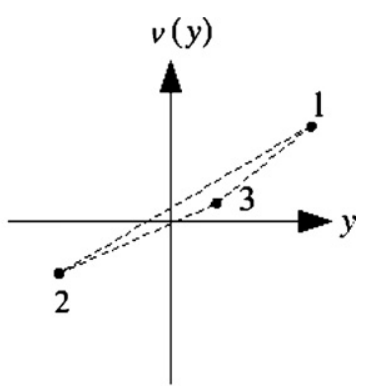

(b)

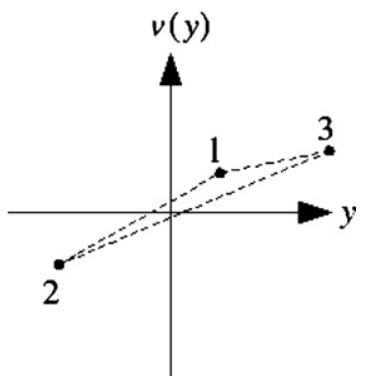

(d)

Figure 3. Cases to be considered for the configuration of the points $\left(f_{i}, v\left(f_{i}\right)\right), i=1,2,3$. (a) The three points are collinear. (b) The point with an intermediate $y$-value (note $y$ is the horizontal co-ordinate here) is below the line joining the other two points. (c) The point with an intermediate $y$-value is above the line joining the other two points, and has the $y$-value less than the average value $f_{\text {av }} \equiv\left(f_{1}+f_{2}+f_{3}\right) / 3$. (d) The point with intermediate $y$-value is above the line joining the other two points, and has the $y$-value greater than or equal to the average value $f_{\mathrm{av}}$. The points have been numbered so that $f_{1}>f_{\mathrm{av}}, f_{2}<f_{\mathrm{av}}$, and removing $f_{3}$ in the procedure outlined in figure 2 will increase the ratchet velocity.

Case illustrated by figure 3(c). Without loss of generality the points may be labelled as shown, that is, the most negative $f$ as ' 3 '; this is the point that will be 'removed'. From the figure, $m_{21}<m_{23}$, and since $f_{3}<f_{2}$ then from (5), $\Delta\langle\dot{x}\rangle>0$.

Case illustrated by figure $3(d)$. Without loss of generality the most positive $f$ may be labelled as ' 3 '. From the figure, $m_{21}>m_{23}$, and since $f_{3}>f_{2}$ it follows that $\Delta\langle\dot{x}\rangle>0$.

Cases $(c)$ and $(d)$ are treated differently so that in each there exists a solution to (4), as discussed in the text below that equation.

Thus replacing the trichotomous (three-valued) region by a dichotomous one will increase the ratchet velocity or at worst keep it the same. In doing so the $N$-valued force has been replaced by an $(N-1)$-valued force. Repetition of the above procedure, at each step increasing the ratchet velocity, will end with a dichotomous force. (Some of these iterations will require operation on segments with unequal width, but the above mathematics can be easily modified to account for this.) As the width of the original discretization $d t$ approaches zero, the above method approaches an optimization of the original waveform $f(t)$.

For any admissible force, then, a dichotomous one with higher velocity can be found. Therefore an optimal force for maximum velocity, if it exists, must be dichotomous. By an analogous argument to the above, another dichotomous force must provide the most negative (minimum) velocity. 
The procedure in this proof will not necessarily converge to the optimum dichotomous waveform, but does always find one that gives a greater velocity than the arbitrary admissible waveform with which it began.

\section{Dichotomous optimal force for the ratchet}

This section finds further properties of the dichotomous, optimal force, assuming that

$$
\text { sign } y \frac{\mathrm{d}}{\mathrm{d} y} \frac{v(y)}{y}>0 \quad \text { for } \quad y \neq 0,
$$

and that $v(y)$ is continuous and smooth. The response (2) satisfies these conditions.

The defining characteristics of a dichotomous force are the force's two values and the time spent at each value. The ratchet velocity $\langle\dot{x}\rangle(f)$ is clearly insensitive to time shifts. Likewise it is insensitive to 'time-mixing' of the force, where it alternates many times between its values, as long as the total time spent at each value is the same. A force with just two transitions per period (the minimum possible: one positive direction, one negative direction), though, is likely to be more experimentally practical and also more likely to preserve adiabaticity of the particle's dynamics.

Define the dichotomous force

$$
f(t)= \begin{cases}M>0, & 0 \leqslant t<T d \\ N<0, & T d \leqslant t<T .\end{cases}
$$

Since the force must have zero mean,

$$
M d+N(1-d)=0 .
$$

The velocity can therefore be written as

$$
\langle\dot{x}\rangle=v(M) d+v\left(-M \frac{d}{1-d}\right)(1-d) .
$$

Setting $0=\frac{\partial\langle\dot{x}\rangle}{\partial d}$, treating $M$ as constant and using constraint (7) to specify $N$ as a function of $d$ gives

$$
v^{\prime}\left(N^{*}\right)=\frac{v(M)-v\left(N^{*}\right)}{M-N^{*}},
$$

where the prime here denotes a derivative. This provides a condition for the locally extremizing $N, N^{*}$. It holds for all response functions for which the optimal force is dichotomous. It can be shown that $\frac{\partial^{2}\langle\dot{x}\rangle}{\partial d^{2}} \propto v^{\prime \prime}(N)$, so the type of the extremum is determined by the sign of $v^{\prime \prime}\left(N^{*}\right)$.

The next step is to attempt optimization with respect to $M$. Return to (8). When forming $\frac{\partial\langle\hat{x}\rangle}{\partial M}$ one must consider $\partial d / \partial M$; however, these terms cancel to give

$$
\frac{\partial\langle\dot{x}\rangle}{\partial M}=d\left[v^{\prime}(M)-v^{\prime}\left(N^{*}\right)\right] .
$$

This quantity, as will now be shown, is greater than zero for all $M>0$. Note first that (9) can be interpreted graphically: the tangent of $v(y)$ at $N^{*}$ is collinear with the secant joining $\left(N^{*}, v\left(N^{*}\right)\right)$ and $(M, v(M))$, as sketched in figure 4. An equation describing this line, to be denoted by $T$, is

$$
v_{T}\left(y_{T}\right)=v^{\prime}\left(N^{*}\right)\left(y_{T}-N^{*}\right)+v\left(N^{*}\right) .
$$

It intercepts the vertical $(v)$ axis at

$$
v_{T}(0)=-v^{\prime}\left(N^{*}\right) N^{*}+v\left(N^{*}\right)>0,
$$

6 


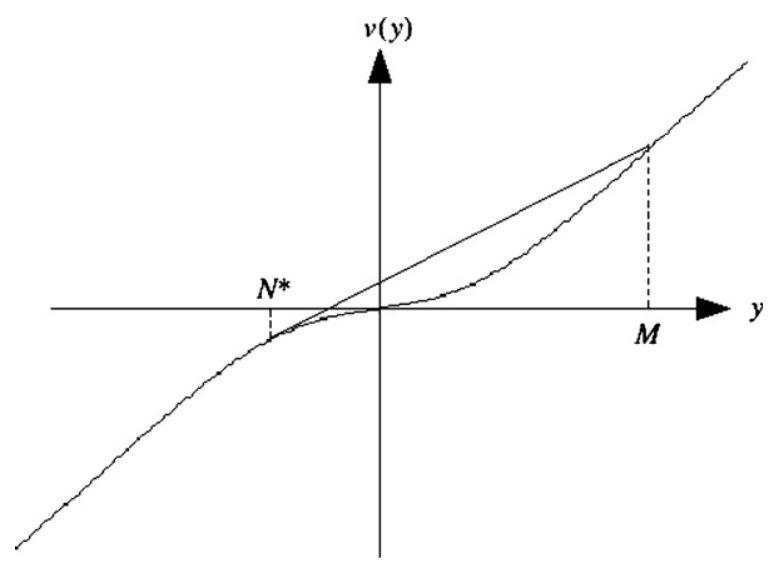

Figure 4. Graphical representation of the condition for $N^{*}(9)$.

with the inequality following from (6) and $N^{*}<0$. Since the line also contains $(M, v(M))$ then another equation describing it is

$$
v_{T}\left(y_{T}\right)=v^{\prime}\left(N^{*}\right)\left(y_{T}-M\right)+v(M) .
$$

According to this equation the $v$-intercept is

$$
v_{T}(0)=-v^{\prime}\left(N^{*}\right) M+v(M) .
$$

Suppose $v^{\prime}(M) \leqslant v^{\prime}\left(N^{*}\right)$. Then

$$
v_{T}(0) \leqslant-v^{\prime}(M) M+v(M)<0
$$

with the last inequality following from (6) and $M>0$. But this contradicts the earlier result. Therefore it follows that in fact $v^{\prime}(M)>v^{\prime}\left(N^{*}\right)$ and by (10) that $\frac{\partial\langle\dot{x}\rangle}{\partial M}>0$ for all $M>0$. Therefore the optimum $M$, for maximum velocity, is its maximum permitted value, $L$.

In order to find the waveform that minimizes the velocity the above argument may be repeated but first optimizing with respect to $M$. It then follows that the optimum $N$ is the most negative possible, $-L$. If $v(y)=-v(-y)$, as does (2) for symmetric $V(x)$, then this force is the negative of the one just found to maximize the velocity.

By inspection of figure 1, it appears that for each $v(y)$ given by (2) there exists exactly one $N^{*}$ that satisfies (9) for fixed $M$, as sketched in figure 4. Further, it appears that $v^{\prime \prime}\left(N^{*}\right)$ is of the correct sign for local maximization (in figure 4) or minimization. I state without proof that this is indeed the case. Since there is only one local extremum in ratchet velocity (as one varies $N$ ) and it is of the correct type, and $v(y)$ is smooth and continuous, it follows that the global extremum is also here.

By theorem 1, then, there exists an optimum force for maximization (and another for minimization), it is dichotomous, and its characteristics are unique.

\section{Numerical work and discussion}

Figure 5 shows the optimal $N^{*}$ and $d$ at various force limits $L$ and temperatures $k_{B} T$. Note that, as intuitively expected from figure 4, for most $L$ at $k_{B} T=0.1$ the optimal $N^{*}$ is on or close to the knee in figure 1 , while at higher temperature $N^{*}$ changes more with $L$. As the upper limit increases, the duty cycle shows that the dichotomous, optimal force spends a smaller fraction of time at this upper value. 


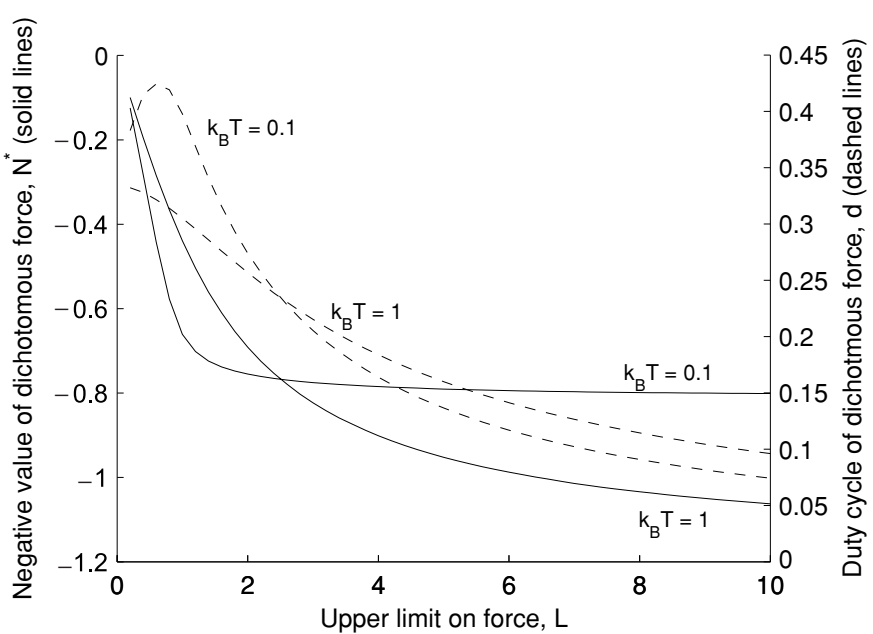

Figure 5. Optimal value for the negative part of the dichotomous force (when maximizing the ratchet velocity), $N^{*}$ (solid lines), and optimal value for the dichotomous force's duty cycle, $d$ (dashed lines), as functions of the limit $L$, for two temperatures $k_{B} T$.

A common ratchet driving waveform is the biharmonic drive,

$$
f_{\text {bihar }}(t)=A \cos \omega t+B \cos (2 \omega t+\phi) .
$$

According to perturbation analysis this will generate, in an overdamped spatially symmetric ratchet, at the limit of small force amplitude, an average ratchet velocity that scales with $A^{2} B \cos \phi[3,14,15]$. The optimal biharmonic phase is therefore $\phi=0$ (or $\phi=\pi$, for transport in the negative direction) and, if the maximum value of the force is limited to $L$, the optimal amplitudes for these phases are $B=L / 3$ and $A=2 L / 3$.

The velocities predicted by (1)-(2) are shown in figure 6 for both the optimal dichotomous force obtained previously and this perturbatively optimal biharmonic force. At small $L$ the biharmonic responses are almost as large as the dichotomous responses, as is appropriate for a perturbation analysis. For larger forces, however, the biharmonic responses are significantly less than the corresponding dichotomous responses, and even decay for very large forces. While the rates of increase of the dichotomous responses do slow, they will show no such turning point, as shown in the paragraph following (10).

For an experimentalist or engineer designing an overdamped ratchet close to the adiabatic limit — or even as a first guess away from the adiabatic limit — and who can freely choose the time dependence of the force up to a limiting value $\pm L$, a dichotomous force, it has been shown, will give the largest possible average velocity. The optimal characteristics of the force can be solved with the equations or approximated as with the graphs given here.

That the optimal force is dichotomous is to be intuitively expected when one notes that the response (1), as should be expected for an adiabatic response, depends only on the force $f$, not any of its derivatives or anti-derivatives. There is no 'penalty', then, for the discontinuities in the dichotomous force.

It is acknowledged that practical restrictions of specific experimental arrangements may in some cases more strongly influence the form of the force, for example biharmonic forces from the mixing of laser beams [16], or near-dichotomous forces from the application of pressure across a membrane [17].

The results for optimization of the driving force also apply for arbitrary potentials $V(x)$, provided the potential is continuous, although the response (2) may break $v(y)=-v(-y)$ 


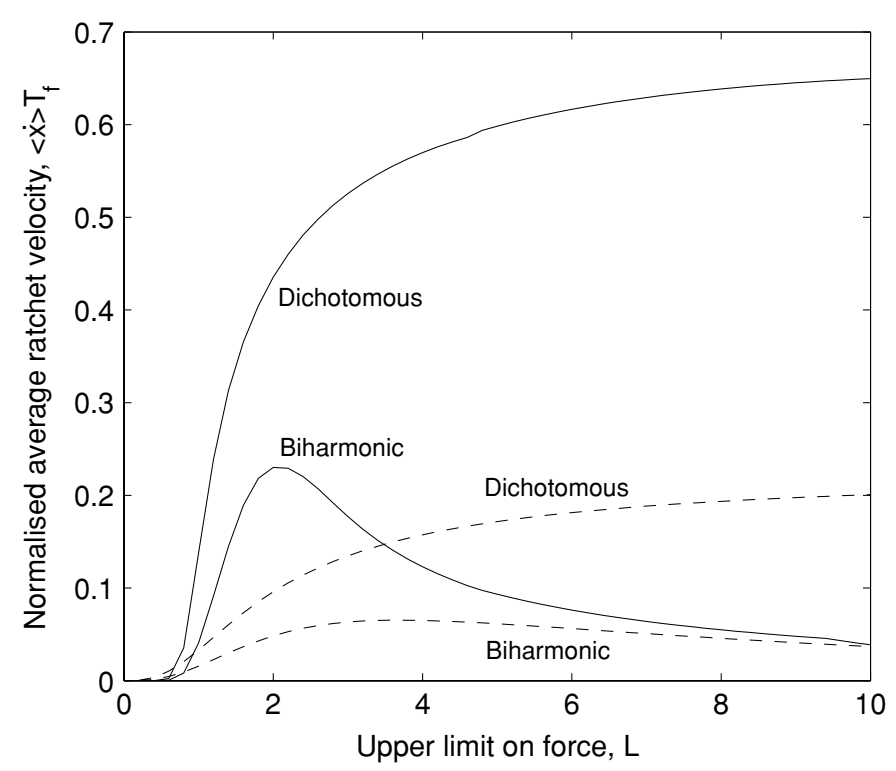

Figure 6. Velocities, normalized by the force's period $T_{f}$, predicted by (1) and (1) for the dichotomous optimal force and the perturbatively optimal biharmonic force with the same maximum value $L$, for temperatures $k_{B} T=0.1$ (solid lines) and 1 (dashed lines).

symmetry. A case with asymmetric $V(x)$ where the optimal force is symmetric, as well as dichotomous, can even be envisioned.

To the author's knowledge, only one study has previously considered the dependence of the ratchet current on the general shape of the potential or force. In an interesting recent article, Chacón [14] proposed a measure of 'degree of symmetry breaking' (DSB) for each of the relevant symmetries, in an effort to quantify the connection between symmetry breaking and ratchet current. The only evidence he showed to support this measure, however, was an interpretation that for a biharmonic driving force it predicted the same optimal combination of amplitudes as the perturbation analysis discussed in section 4. Furthermore, the measures diverge for a wide range of parameters, while any DSB, if it is to be correlated with ratchet current, should presumably remain finite. (The Cauchy principal value of the DSBs do exist, but, it can be shown, at least one is constant for all biharmonic forces.) Considering the connection to ratchet current that motivated the DSBs, perhaps the most appropriate measure of DSB for the present system is the predicted ratchet current (1)-(2).

\section{Conclusions and future work}

An optimum force for an overdamped, adiabatic ratchet, amongst the admissible forcesbriefly, forces with zero mean and maximum absolute value not greater than $L$-if it exists, is dichotomous. The optimum characteristics of the dichotomous force, for maximum velocity, are (any time-shifted or time-mixed version of this force will give the same velocity)

where $N^{*}<0$ is a solution of

$$
f^{*}(t)= \begin{cases}L, & 0 \leqslant t<d \\ N^{*}, & d \leqslant t<T,\end{cases}
$$

$$
v^{\prime}\left(N^{*}\right)=\frac{v(L)-v\left(N^{*}\right)}{L-N^{*}}
$$


and $d$ is then given by $d=N^{*} /\left(N^{*}-L\right)$. From plots of the response (2), it appears that a (unique) dichotomous optimal force does exist, though this was not formally proven.

The main result of this paper, theorem 1, may apply to a wide range of problems. It was shown that for any response of the form $\frac{1}{T} \int_{0}^{T} v(f(t)) d t$, where $f(t)$ has a finite number of discontinuities, has a zero mean and a size constrained by its maximum absolute value, the response is extremized by a dichotomous force.

The result may be useful for engineers designing overdamped Brownian motors in the adiabatic limit, or even as a first guess away from the adiabatic limit, wishing to achieve the maximum possible average velocity from the ratchet. For large forces a dichotomous force can generate a much larger velocity than that generated by the biharmonic force optimized by perturbation analysis.

There remain many ratchet configurations in which this optimization task could be addressed. For the case of an overdamped, adiabatic ratchet with time-symmetric force, early results indicate that the optimum potential may be close to a sawtooth waveform, which would be a pleasingly complementary result to the above. One could consider an overdamped rocking ratchet with fast driving, from which some conclusions about the optimal waveform for all driving frequencies might be drawn. There are also the underdamped, both adiabatic and fast-driven, cases. Finally, one could consider optimization with respect to other measures such as efficiency, Péclet number or stall force. In these analyses, more sophisticated methods such as the calculus of variations or Pontryagin's maximum principle [18] may be useful.

\section{References}

[1] Feynman R P, Leighton R B and Sands M 1963 The Feynman Lectures on Physics vol 1 (Reading, MA: Addison-Wesley)

[2] Smoluchowski M V 1912 Experimentell nachweisbare, der üblichen Thermodynamik widersprechende Molekularphänomene Phys. Z. 131069

[3] Denisov S, Flach S, Ovchinnikov A A, Yevtushenko O and Zolotaryuk Y 2002 Broken space-time symmetries and mechanisms of rectification of ac fields by nonlinear (non)adiabatic response Phys. Rev. E 66041104

[4] Denisov S, Morales-Molina L, Flach S and Hänggi P 2007 Periodically driven quantum ratchets: symmetries and resonances Phys. Rev. A 75063424

[5] Peskin C S, Odell G M and Oster G F 1993 Cellular motions and thermal fluctuations-the Brownian ratchet Biophys. J. 65 316-24

[6] Spudich J A 1994 How molecular motors work Nature 372 515-8

[7] Jülicher F, Ajdari A and Prost J 1997 Modeling molecular motors Rev. Mod. Phys. 69 1269-81

[8] Reimann P 2002 Brownian motors: noisy transport far from equilibrium Phys. Rep. 361 57-265

[9] Hänggi P, Marchesoni F and Nori F 2005 Brownian motors Ann. Phys. 14 51-70

[10] Astumian R Dean and Hänggi P 2002 Brownian motors Phys. Today 55 33-9

[11] Sokolov I M 2001 Irreversible and reversible modes of operation of deterministic ratchets Phys. Rev. E 63 021107

[12] Linke H, Downton M T and Zuckermann M J 2005 Performance characteristics of Brownian motors Chaos 15026111

[13] Gomez-Marin A and Sancho J M 2006 Tight coupling in thermal Brownian motors Phys. Rev. E 74062102

[14] Chacón R 2007 Optimal control of ratchets without spatial asymmetry J. Phys. A: Math. Theor. 40 F413-9

[15] Marchesoni F 1986 Harmonic mixing signal: doubly dithered ring laser gyroscope Phys. Lett. A 119 221-4

[16] Schiavoni M, Sanchez-Palencia L, Renzoni F and Grynberg G 2003 Phase control of directed diffusion in a symmetric optical lattice Phys. Rev. Lett. 90094101

[17] Matthias S and Müller F 2003 Asymmetric pores in a silicon membrane acting as massively parallel Brownian ratchet Nature 424 53-7

[18] Pontryagin L S, Boltyanskii V G, Gamkrelidze R V and Mischchenko E F 1962 The Mathematical Theory of Optimal Processes (New York: Wiley) 